\title{
4-Bromoctavalen und zwei (Brommethylen)homobenzvalene anstelle eines erwarteten Bromoctabisvalens
}

\author{
Manfred Christl ${ }^{*}$, Rainer Nusser und Clemens Herzog \\ Institut für Organische Chemic der Universität Würzburg, \\ Am Hubland, D-8700 Würzburg
}

Eingegangen am 7. August 1987

\begin{abstract}
Die Reaktion von Dibromcarben mit 5-Methoxyhomobenzvalen (5) ergab das trans-Addukt 6 , das sich durch lodtrimethylsilan in Gegenwart von Trietbylamin in den Trimethylsilylether 7 umwandeln lieB. Daraus wurde durch Kaliumcarbonat in Methanol 8,8-Dibromtetracyclo[5.1.0.0.0.4. $0^{3.5}$ ]octan-exo-6-ol (8) erhalten. Das auf dem üblichen Weg dargestellte Methansulfonat 9 von.8 ging mit Alkyllithiumverbindungen nicht in das erwartete Bromoctabisvalen 11 über, sondern lieferte ein Gemisch aus dem Bromoctavalen 12 und den (Brommethylen)homobeazvalenen 14.
\end{abstract}

\section{Bromoctavalene and Two (Bromoriethylene)topnohenzirileves Instiand of in Expected Bromovetubiovialene}

The reaction of dibromocarbene with 5-methoxyhomolbenzvalene (5) gave the trans adduct 6 , which could be converted into the trimethylsilyl ether 7 with iodotrimethylsilane in the presence of triethylamine. Treatment of 7 with potassium carbonate in methanol afforded 8,8-dibromotetracyclo[5.1.0,023., $0^{3,5}$ octan-exa-6-01 (8). Prepared from 8 in the usual manner, the methanesulfonate 9 could not be transformed to the expected bromooctabisvalene 11 with alkyllithium compounds. Instead, a mixture of bromooctavalene 12 and the (bromomethylene)homobenzvalenes 14 was formed.

clopropan-Einheit) zuordnen. Aufgrund bekannter Dichlorcyclopropanierungen von cyclischen Allylalkoholen ${ }^{6)}$ kam dieser Befund nicht überraschend.

Jedoch nahm der Methylether $5^{4}$, dessen Darstellung wir verbessert haben, Dibromcarben zum trans-Addukt 6 auf (67\% Ausbeute). Die herkömmlichen Reagenzien zur Etherspaltung (Halogenwasserstoffsäuren, Borhalogenide) eignen sich für 6 nicht, da sie das Bicyclobutan-System zerstören ${ }^{7}$. Trimethylsilyliodid in Gegenwart von Triethylamin, von Jung und Lyster ${ }^{8)}$ zur Etherspaltung eingeführt, bewerkstelligte aber die gewünschte Reaktion. Dabei entstand der Trimethylsilylether 7 mit ca. $65 \%$ und daraus durch Methanolyse in Gegenwart von Kaliumcarbonat der Alkohol 8 mit quantitativer Ausbeute. Die Struktur von 6-8 wird durch dic NMR-Spektren belegt (Tab. 1). Die trans-Orientierung der Sauerstoffunktionen zur DibromcyclopropanEinheit entnehmen wir aus der nicht aufgelösten Kopplungskonstante $J_{6.7}$.

Nachdem es nicht gelang, das Tosylat von $8 \mathrm{zu}$ isolieren, vermutlich weil das Tosylat-Ion leicht abdissoziiert und dann das Carbokation umlagert, erhielten wir mit Hilfe von Methansulfonylchlorid aus 8 das Mesylat 9 und damit eines der angestrebten Substrate (ca. 35\% Ausbeute). Dessen Behandlung mit Lithiumorganylen bei Temperaturen zwischen -70 und $0^{\circ} \mathrm{C}$ brachte jedoch nicht das erhoffe Bromoctabisvalen 11 hervor, sondern Gemische des bekannten Bromoctavalens $12^{91}$ mit den beiden (Brommethylen)homobenzvalenen 14. Bei $0^{\circ} \mathrm{C}$ in Tetrahydrofuran mit Methyllithium in Ether lag die Ausbeute bei ca. $65 \%$ und das Verhältnis 12:14a:14b bei 1.2:1.0:1.3. Daneben fand sich noch eine geringe Menge Phenylacetylen, dessen Genese in Analogie zur Bildung von 5-Ethinyl-1,3-cyclohexadien aus 8,8-Dibromtetracyclo[5.1.0.0.2.4. $\left.0^{3.5}\right]$ octan und Methyllithium zu sehen ist ${ }^{7,00}$. Die Struktur von 14a,b leiten wir aus den




NMR-Spektren (Tab. 2) ab, wobei für die Zuordnung der Konfiguration die nur bei einem Isomeren aufgelöste Kopplungskonstante $J_{3,8}=1.8 \mathrm{~Hz}$ entscheidend ist, denn nur bei 14a bilden die Bindungen zwischen diesen Protonen einen Zickzack-Weg.

Tab. 1. NMR-spektroskopische Daten von 6-9 in $\mathrm{CDCl}_{3}$

A. 'H-Crentsche versenicoungen (6-Werte) und Mi:tipliz:taten

\begin{tabular}{|c|c|c|c|c|c|c|c|}
\hline$m$ & $\therefore-H$ & 2.H & $3-\mathrm{H}$ & 1-H & $5-\mathrm{H}$ & $6-H$ & $7-H$ \\
\hline 6 ) & br.0d 1.81 & $=0 \mathrm{da} 2.86$ & ctd 1.16 & d:d 2.07 & q qu: 2.54 & or. c 3.23 & br.d:.59 \\
\hline $7^{31}$ & br.od 1.75 & 2002.75 & etd 1.69 & otc $: .97$ & $=$ qu: 2.31 & be. 03.60 & br.d 1.45 \\
\hline$g^{c)}$ & or. $d d 1.8 t$ & $\dot{x} \mathrm{dq} 2.82$ & Dr.ct 1.68 & br.dt 2.10 & $=Q$ Q i i 2.50 & br.dd 3.61 & br.d 1.59 \\
\hline (c) & m 1.92 & $\approx d a 2.9 !$ & $m: .92$ & br.dt 2.24 & $\approx q u 12.70$ & br.c 4.65 & or.d 1.78 \\
\hline
\end{tabular}

a) 3.44 (s, $\mathrm{OCH}_{3}$ ): die Zuorching aller Stgraie ist ourch voE-Messung (Sattiging cer $\mathrm{XH}_{3}$ -

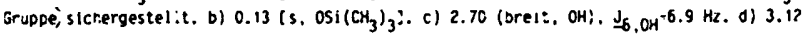
(s. $\mathrm{OSO}_{2} \mathrm{CH}_{3}$ ).

\begin{tabular}{|c|c|c|c|c|c|c|c|c|}
\hline & & $\begin{array}{l}\text { Mit:e: } \\
\text { Abmele? }\end{array}$ & 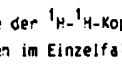 & $\begin{array}{l}\text { :ungskonstante } \\
\text { max:ma! }=0.2\end{array}$ & $\begin{array}{l}\text { en }(\mathrm{Hz}) \text { : } \\
\mathrm{Hz}\end{array}$ & & & \\
\hline ل1.2 & $\underline{\jmath}_{1,4}$ & $\mathrm{~J}_{1.7}$ & $J_{2,3}=J_{3,5}$ & $\underline{J}_{2,4}=J_{4,5}$ & 3.5 & $\mathrm{~J}_{3,4}$ & $\mathrm{~J}_{3,6}$ & $\underline{J}_{5,6}$ \\
\hline 4.3 & 0.8 & $: 0.9$ & 2.4 & 3.3 & 2.9 & 10.0 & 0.8 & 2.4 \\
\hline
\end{tabular}

C. ${ }^{13}$ C-Oenische Verschiejungen (8-Herte) und bef 8

${ }^{13} \mathrm{C}-{ }^{7} \mathrm{H}$-kopplungskonstanten uber ene Bindung ( $\mathrm{HZ}$, zweite $2 \mathrm{el}(\mathrm{l})$

\begin{tabular}{|c|c|c|c|c|c|c|c|c|c|}
\hline Nr. & C: & c. 2 & c. 3 & $c-4$ & C 5 & $C-6$ & C-7 & $C-8$ & Subs:ituent \\
\hline $6^{31}$ & 22.66 & 37.25 & $0.2:$ & 6.05 & 39.79 & 67.45 & 27.28 & $34.5 ?$ & 56.51 \\
\hline 7 & 22.93 & 31.09 & 0.34 & 6.65 & 42.80 & 59.35 & 30.63 & 34.65 & 0.19 \\
\hline \multirow[t]{2}{*}{8} & 22.56 & 37.19 & -0.86 & $7.0:$ & 42.6: & 58.35 & 30.02 & 33.89 & - \\
\hline & 170 & $: 61$ & $2: 0$ & 213 & 158 & $: 49$ & 171 & - & \\
\hline 9 & 21.98 & 36.57 & 0.34 & 6.92 & $40 .: 3$ & 69.36 & $27 .: 2$ & 31.95 & 38.72 \\
\hline
\end{tabular}

a) Jie Zuordning al ler Signale wurde durch $2{ }^{2}{ }^{13} \mathrm{C}$. ${ }^{1} \mathrm{H}$-Korrelation sichergeste:it.<smiles>COC1C(Br)C2C3CC(C3Br)C12</smiles><smiles>BrC1=CC2C3C=CC3C2C1</smiles>

12

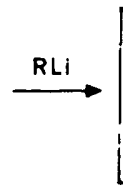

$+-\mathrm{CH}_{3} \mathrm{SO}_{3}$

13

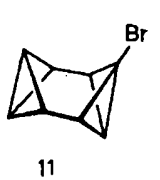

11

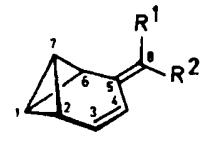

14a: $R^{1}-H, R^{2}=B r$ $b: R^{i}=B r, R^{2}-H$
Tab. 2. NMR-spektroskopische Daten von $14 \mathrm{a}, \mathrm{b}$ in $\mathrm{CDCl}_{3}$

A. 'H-Cherische Verschiebungen (O-Kerte) $)^{d}$, Multiplizitaten und Kogplungskorstanter: (Az)

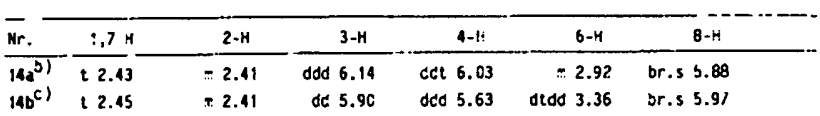


$\underline{J}_{2,4}=1.0, \underline{J}_{3,4} \quad 9.7, J_{3,8}=1.8, \underline{J}_{4,6}=2.0, \dot{u}_{4,8}=\left(. \bar{c}\right.$. c) $\dot{s}_{-1.2}=\underline{J}_{1,6}=2.5, \underline{J}_{2.3} ;$

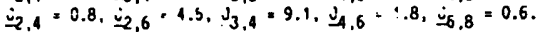

B. ${ }^{13} \mathrm{C}$-Chemische Verscriebingen (o-werie)

\begin{tabular}{|c|c|c|c|c|c|c|c|}
\hline$\ddot{k r}$. & $c-1,7$ & $C-2$ & C. 3 & $c-4$ & $c .5$ & $c-6$ & $c-8$ \\
\hline 14. & 12.24 & 33.70 & $\left(: 33.32^{\mathrm{d}} \overline{(}\right.$ & $\left(17.99^{a)}\right.$ & D) & $43 .: 0$ & 99.53 \\
\hline 146 & 11.99 & 34.00 & $\left(28.87^{\mathrm{d}}\right)$ & $\left(20.40^{8)}\right.$ & b) & 40.07 & 103.26 \\
\hline
\end{tabular}

d) Zworctung unsicher. o) Wegen zu geringer intensita: r:ch: becbecritet.
Daß 9 kein 11 liefert, dürfte an der ausschließlichen exoOrientierung des Lithiumatoms in 10 liegen. Obwohl in 7Chlor- und 7-Brom-7-lithionorcaran bei Gegenwart von 7,7Dichlor- bzw. -Dibromnorcaran der Platztausch von Halogen- und Lithiumatom leicht vonstatten geht ${ }^{11}$, scheint eine derartige Äquilibricrung in $\mathbf{1 0}$ in der Konkurrenz gegen dic neuartige Fragmentierung zum Allylcarben 13 nicht zum Zuge zu kommen. Die Bildung der Produkte 12 und 14 kann von 13 aus zwanglos durch CC- bzw. CH-Insertion des Carbenzentrums erklärt werden. Ein zu 12 analoges Brombutadien entsteht möglicherweise auf dem gleichen Wege neben 1-Brom-2,2-dimethylbicyclo[1.1.0]butan bei der Umsetzung von 1,1-Dibrom-3-(chlormethyl)-2,2-dimethylcyclopropan mit Methyllithium ${ }^{2}$.

Wir danken der Deutschen Forschungsgemeinschaft und dem Fonds der Chemischen Industrie für die Förderung und Herrn Diplomchemiker A. Kraft für die NOE-Messung und die $2 \mathrm{D}-{ }^{13} \mathrm{C},{ }^{1} \mathrm{H}$ Korrelation.

\section{Experimenteller Teil}

${ }^{1} \mathrm{H}$ - und ${ }^{13} \mathrm{C}-\mathrm{NMR}$ : Bruker WM 400 und AC 200. - IR: Beckman AccuLab 4. - MS: Varian MAT CH 7. - Elementaranalysen: Carlo Erba Strumentatione Elemental Analyzer 1106. Schmp.: Heiztischmikroskop nach Kofler, Optische Werke Reichert (Wien).

Die NOE-Messung und die $2 \mathrm{D}-{ }^{13} \mathrm{C},{ }^{1} \mathrm{H}-$ Korrelation von 6 wurden am Bruker AC 200 ausgeführt. Für das NOE-Differenz-Spektrum wurde die Probe durch drei Einfrieren-Abpumpen-AuftauenCyclen von Sauerstoff befreit und i. Vak. abgeschmolzen. Bei der $2 \mathrm{D}-{ }^{13} \mathrm{C},{ }^{\prime} \mathrm{H}$-Korrelation wurde das Bruker-Standardprogramm XHCORR. AU benutzt.

5-Methoxytricyclo[4.1.0.0.7 Jhept-3-en (5-Methoxyhomobenzvalen, 5): Die publizierte Synthese ${ }^{4)}$ wurde optimiert. Insbesondere entfällt jetzt die aus Sicherheitsgründen ${ }^{12)}$ aufwendige Isolierung von 7,7-Dibromtetracyclo[4.1.0.0.4.4. $0^{3.5}$ ]heptan. - GemäB unserer Vorschrift ${ }^{13)}$ addierte man Dibromcarben an $50.6 \mathrm{~g}(648 \mathrm{mmol})$ Benzvalen und arbeitete auf, bis die trockene Lösung von 7,7-Dibromtetracyclo[4.1.0.0.2, $0^{3,5}$ ]heptan mit allen Verunreinigungen in Ether/Pentan vorlag. Dieses Rohprodukt tropfte man zu einer Lösung von $74 \mathrm{~g}$ (3.2 mol) Natrium in $900 \mathrm{ml}$ Methanol, destillierte Ether und Pentan vollständig ab und kochte dann $36 \mathrm{~h}$ unter Rückfluß. Nach dem Abkühlen hydrolysierte man, verdampfte den größten Teil des Methanols i. Vak., extrahierte zuerst mit $300 \mathrm{ml}$ und dann dreimal mit je $70 \mathrm{ml}$ Ether, trocknete die vereinigten Etherphasen mit $\mathrm{Na}_{2} \mathrm{SO}_{4}$ und engte i. Vak. ein. Aus dem Rückstand wurden durch Vakuumdestillation ${ }^{4} 73.7 \mathrm{~g}$ (57\%) 4-Brom-5methoxytricyclo[4.1.0.0 $0^{2,7}$ hept-3-en als gelbe Flüssigkeit gewonnen. -. Die Cmwandlung dieser Bromverbindung in 5 gemä $B$ Lit. ${ }^{4)}$ gelingt jetzt mit $92 \%$ Ausbeute.

8.8-Dibrom-exo-6-methoxytetracyclo( $5.1 .0 .0^{2.4} .0^{3.5}$ Joctan (6): Unter Stickstoff wurden bei -15 bis $-20^{\circ} \mathrm{C} 6.85 \mathrm{~g}$ (27.1 mmol) Bromoform innerhalb von $30 \mathrm{~min}$ zur gerührten Suspension von $4.56 \mathrm{~g}$ (40.6 mmol) Kalium-tert-butoxid in $1.64 \mathrm{~g}(13.4 \mathrm{mmol}) 5$ und $40 \mathrm{ml}$ Petroleumbenzin $\left(50-70^{\circ} \mathrm{C}\right)$ getropft. Man ließ dann auf $20^{\circ} \mathrm{C}$ erwärmen und rührte weitere $30 \mathrm{~min}$. Nach Hydrolyse und Phasentrennung extrahierte man die wäßrige Phase dreimal mit je $20 \mathrm{ml}$ Petroleumbenzin, trocknete die vereinigten organischen Phasen mit $\mathrm{Na}_{2} \mathrm{SO}_{4}$ und engte i. Vak. cin. Aus dem Rückstand destillierten bei $50-60^{\circ} \mathrm{C}(\mathrm{Bad}) / 0.001$ Torr $2.65 \mathrm{~g}(67 \%) 6$ als farbloses Öl. -- MS (70 eV): $m / z(\%)=231(37), 229(77), 227(40), 134(100)$, 
$102(37), 91$ (78), 77 (33), 65 (39), 51 (46), 39 (65). - ' $\mathrm{H}$ - und ${ }^{13} \mathrm{C}-$ NMR: Tab. 1.

$$
\begin{array}{llllll}
\mathrm{C}_{9} \mathrm{H}_{10} \mathrm{Br}_{2} \mathrm{O} \text { (294.0) } & \text { Ber. C } 36.77 & \text { H } 3.43 & \mathrm{Br} 54.36 \\
& \text { Gef. C } 37.09 & \text { H } 3.49 & \text { Br } 54.38
\end{array}
$$

8,8-Dibrom-exo-6-(trimethylsiloxy)tetracyclo[5.1.0.0.0.4. $0^{3.5}$ /octan (7): Unter Stickstoff und Rühren gab man $3.20 \mathrm{ml}(4.73 \mathrm{~g}, 23.6$ mmol) lodtrimethylsilan zur Lösung von $5.30 \mathrm{~g}$ (18.0 mmol) 6 und $3.29 \mathrm{ml}(2.39 \mathrm{~g}, 23.6 \mathrm{mmol})$ Triethylamin in $10 \mathrm{ml}$ absol. Chloroform. Man bewahrte das Gemisch $15 \mathrm{~d}$ bei $20^{\circ} \mathrm{C}$ auf, filtrierte dann den entstandenen Niederschlag $a b$ und engte das Filtrat i. Vak. cin. Aus dem Rückstand destillierten bei $50-70^{\circ} \mathrm{C}(\mathrm{Bad}) / 0.001$ Torr $5.10 \mathrm{~g}(80 \%)$ eines farblosen Ols. Dic gesamte Aufarbeitung erfolgte unter Stickstoff. Das 'H-NMR-Spektrum wies als Hauptkomponente des Produkts den Trimethylsilylether 7 aus. Dancben lag mit einem Anteil von ca. $20 \%$ eine Verbindung mit $7 . u$ denen von $7 \mathrm{schr}$ ähnlichen Banden vor. Insbesondere gleicht das br. $d$ bei $\delta 3.72$ in Aufspaltung und Linienbreite dem von 7 bci 3.60. Die Elementaranalyse wurde durch diese Verunreinigung nicht beeinträchtigt. MS $(70 \mathrm{cV}): m / z(\%)=354,352,350\left(0.2,0.4,0.2, \mathrm{M}^{+}\right), 289(2)$. 287 (4), 285 (2), 192 (7), 103 (7), 91 (8), 75 (11), 45 (9). - 'H- und ${ }^{13} \mathrm{C}$-NMR: Tab. 1.

$$
\begin{array}{lll}
\mathrm{C}_{11} \mathrm{H}_{16} \mathrm{Br}_{2} \mathrm{OSi} \text { (352.2) } & \text { Ber. C } 37.52 \mathrm{H} 4.58 \\
& \text { Gef. C } 37.80 \mathrm{H} 4.51
\end{array}
$$

8.8-Dibromtetracyclo/ 5.1.0.0.0.4. $0^{3.5}$ Joctan-exo-6-ol (8): $2.06 \mathrm{~g}(5.85$ mmol) 7 wurden bei $20^{\circ} \mathrm{C} 30 \mathrm{~min}$ mit ca. $50 \mathrm{mg} \mathrm{K}{ }_{2} \mathrm{CO}_{3}$ in $50 \mathrm{ml}$ Methanol gerührt. Man verdampfte dann das Solvens i. Vak., nahm den Rückstand mit $20 \mathrm{ml}$ Ether auf, wusch $\mathrm{mit} 30 \mathrm{ml}$ Wasser, trennte die Phasen, extrahierte die wäßrige Phase zweimal mit je $20 \mathrm{ml}$ Fther und trocknete die vercinigten organischen Phasen mit $\mathrm{Na}_{2} \mathrm{SO}_{4}$. Nach Verdampfen des Ethers i. Vak. erhielt man $1.63 \mathrm{~g}$ (quantitativ) eines farblosen Ols durch Destillation bei $80-85^{\circ} \mathrm{C}$ (Bad)/0.001 Torr. Laut 'H-NMR-Spektrum lag als Hauptkomponente 8 vor und mit einem Anteil von ca. $20 \%$ ein zweites Produkt, dessen Banden denen von 8 ähnlich sind. Insbesondere gleicht das Signal bei $\delta 3.78 \mathrm{dem}$ von 8 bei 3.67 in Form und Aufspaltung. Dic Elementaranalyse wurde durch diese Verunreinigung nicht beeinträchtigt. - IR (Film): $3340 \mathrm{~cm}^{-1}$ (sehr breit, OH). - MS (70 eV): $m / z(\%)=282,280,278\left(0.3,0.8,0.4, M^{+}\right), 217(12), 215(26), 213$ (15), 121 (25), 120 (36), 92 (38), 91 (100), 66 (47), 65 (41), 51 (33), 39 (51). - ' $\mathrm{H}$ - und ${ }^{13} \mathrm{C}-\mathrm{NMR}$ : Tab. 1.

$\mathrm{C}_{8} \mathrm{H}_{8} \mathrm{Br}_{2} \mathrm{O}(280.0)$ Ber. C 34.32 H 2.88 Gef. C 34.06 H 2.92

p-Nitrobenzoat von 8: Darstellung wie üblich mit Hilfe von 4Nitrobenzoylchlorid in Pyridin (52\% Ausb.), Schmp. $125-128^{\circ} \mathrm{C}$ nach Umlösen aus Chloroform/Hexan.

$$
\begin{array}{lllll}
\mathrm{C}_{55} \mathrm{H}_{11} \mathrm{Br}_{2} \mathrm{NO}_{4} \text { (429.1) } & \text { Ber. C } 41.99 & \text { H } 2.59 & \text { N } 3.26 \\
& \text { Gef. C } 41.71 & \text { H } 2.51 & \text { N } 3.20
\end{array}
$$

Methansulfonat 9 von 8: Unter Stickstoff legte man $2.00 \mathrm{~g}$ (7.14 mmol) 8 und $3.06 \mathrm{ml}(21.4 \mathrm{mmol})$ Triethylamin in $40 \mathrm{ml}$ Dichlormethan vor, kühlte auf $-30^{\circ} \mathrm{C}$ und tropfte unter Rühren innerhalb von $15 \mathrm{~min} 0.62 \mathrm{ml}$ (899 $\mathrm{mg} .7 .85 \mathrm{mmol})$ Methansulfonylchlorid zu. Nach Aufwärmen auf $0^{\circ} \mathrm{C}$ rührte man noch $4 \mathrm{~h}$, goß das Gemisch dann in $60 \mathrm{ml}$ Eiswasser, trennte die Phasen, wusch die organische Phase zweimal mit je $30 \mathrm{ml}$ Eiswasser, trocknete sic (einige min Rühren mit $\mathrm{Na}_{2} \mathrm{SO}_{4}$ ) und verdampfte das Solvens bei $0^{\circ} \mathrm{C} / 20$ Torr. Der braune ölige Rückstand wurde mit kaltem Ether behandelt. Nach Filtricren und Abdampien des Ethers bei $0^{\circ} \mathrm{C}^{\prime} 20$ Torr blieb cin gelbes Ol zurück, aus dem durch Zugabe von kaltem Pentan ein gelber Feststoff mit Schmp. 65-68 ${ }^{\circ} \mathrm{C}$ ausgefällt wurde. Die gesamte Aufarbeitung erfolgtc zügig. Aufgrund der NMR-Spektren handelte es sich dabei in der Hauptsache um 9 mit einem Anteil von ca. $30 \%$ an unbckannten Verunrcinigungen. Bci $-25^{\circ} \mathrm{C}$ kann
9 einige Monate aufbewahrt werden, zersetzt sich aber bei $20^{\circ} \mathrm{C}$ innerhalb von $24 \mathrm{~h}$. $-\mathrm{MS}(70 \mathrm{eV}): m / z(\%)=360,358,356(0.5$, $\left.1.0,0.5, \mathrm{M}^{+}\right), 265(11), 263(22), 261(12), 184(36), 183(38), 182(38)$, $181(35), 121(21), 119(32), 103$ (100), 102 (71), 91 (57), 90 (25), 79 (43), 77 (59), $65(30), 63(26), 57$ (27), 51 (57), 50 (25), $39(42)$. - ' ${ }^{1} \mathrm{H}$ und ${ }^{13} \mathrm{C}$-NMR: Tab. 1.

$$
\begin{array}{llll}
\mathrm{C}_{9} \mathrm{H}_{10} \mathrm{Br}_{2} \mathrm{O}_{3} \mathrm{~S} \text { (358.1) } & \text { Ber. C } 30.19 \text { H } 2.82 \\
& \text { Gef. C } 31.68 \text { H } 3.14
\end{array}
$$

Reaktion von Methyllithium mit dem Methansulfonat 9: Unter Stickstoff und Rühren tropfte man innerhalb von $30 \mathrm{~min} 0.63 \mathrm{mmol}$ Methyllithium $(0.49 \mathrm{ml} 1.3 \mathrm{~N}$ in Ether $) \mathrm{zu} 150 \mathrm{mg}(0.42 \mathrm{mmol}) 9$ in $7 \mathrm{ml}$ Tetrahydrofuran, wobei die Temperatur des Gemisches auf $0^{\prime \prime} \mathrm{C}$ gchalten wurde. Nach $4 \mathrm{~h}$ weitcren Rührens bei $0^{\circ} \mathrm{C}$ gab man $20 \mathrm{ml}$ Wasser zu, trenntc die Phasen, extrahierte dic wäBrige Phase zweimal mit je $10 \mathrm{ml}$ Ether, trocknete die vereinigten organischen Phasen mit $\mathrm{Na}_{2} \mathrm{SO}_{4}$ und engte i. Vak. ein. Aus dem Rückstand destillierten bei $20^{\circ} \mathrm{C} / 0.001$ Torr (Kühlung der Vorlage mit Trokkeneis) $50 \mathrm{mg}(65 \%)$ einer farblosen Flüssigkeit. Ihre NMR-Spektren charakterisicrten dic Hauptkomponenten als 4-Bromoctavalen $(12)^{9 /}$ sowic die beiden 5-(Brommethylen) tricyclo $\left[4.1 .0 .0^{2.7}\right.$ /hept-3ene $14 \mathrm{a}$ und $14 \mathrm{~b}$ ( $\mathrm{Tab}$. 2). Von den in geringer Menge vorhandenen weiteren Bestandteilen wurde Phenylacetylen identifiziert, und zwar durch Zusatz einer kleinen Menge der authentischen Substanz, wodurch bei erneuter Aufnahme des Spektrums die betreffenden Banden verstärkt auftraten. Das Verhältnis 12:14a:14b: Phenylacetylen lag bei $7: 6: 8: 1$. Bei Verwendung von $n$-Butyllithium anstelle von Methyllithium entstanden die gleichen Produkte; bei $-70^{\circ} \mathrm{C}$ trat Phenylacetylen nicht auf, und das Vcrhältnis 12:14a:14b war $2: 1: 1$.

\section{CAS-Registry-Nummern}

5: 70205-25-3 / 6: 110472-25-8 / 7: 110472-26-9 / 8: 110472-27-0/ 8-p-Nitrobenzoat: 110472-28-1 / 9: 110472-29-2/11: 110472-32-7 / 12: $103148-64-7$ / 14a: 1 10472-30-5 / 14b: 110472-31-6 / 7,7-Dibromtetracyclo[4.1.0.0. $0^{2.4} 0^{3.5}$ ] heptan: 50399-12-7 / 4-Brom-5-methoxytricyclo[4.1.0.0 $0^{2,7}$ hept-3-en: 59574-44-6 / Benzvalen: $659-85-8$

${ }^{11}$ C. Rücker, H. Prinzbach, Angew. Chem. 97 (1985) 426; Angew. Chem. Int. Ed. Engl. 24 (1985) 411; C. Rücker, H. Prinzbach, H. Irngartinger, R. Jahn, H. Rodewald, Tetrahedron Lett. 27 (1986) 1565; T. Netscher, R. Schwesinger, B. Trupp, H. Prinzbach, ibid. 28 (1987) 2115.

2) N. O. Nilsen, L. Skattebøl, M. S. Baird, S. R. Buxton, P. D. Slowcy, Tetrahedron Lett. 25 (1984) 2887.

3) 3a) A. Düker, G. Szeimics, Tetrahedron Lett. 26 (1985) 3555. 3b) K. Semmler, G. Szeimies, J. Belzncr, J. Am. Chem. Soc. 107 (1985) 6410. - 3c) A. Düker, Dissertation, Lniv. München 1986; wir danken Prof. Szeimies für die Mitteilung dieser Ergebnisse.

4) M. Christl, C. Herzog, D. Brückner, R. Lang, Chem. Ber. 119 (1986) 141.

5) M. Christl, G. Freitag, G. Brüntrup, Chem. Ber. 111 (1978) 2320.

${ }^{6}$ F. Mohamadi, W. C. Still, Tetrahedron Iett. 27 (1986) 893.

"Vgl. die Thermolyse von 8,8-Dibromtetracyclo[5.1.0.0.4.4. 0.5 octan und seine Reaktion mit $\mathrm{Et}_{2} \mathrm{O}-\mathrm{BF}_{3}: \mathrm{M}$. Christl, M. Lechner, Chem. Ber. 115 (1982) 1.

8) M. E. Jung, M. A. Lyster, J. Org. Chem. 42 (1977) 3761.

9) M. Christl, R. Lang, C. Herzog, Tetrahedron 42 (1986) 1585.

10) M. Christl, M. Lechner, Angew. Chem. 87 (1975) 815; Angew. Chem. Int. Ed. Engl. 14 (1975) 765; L. A. Paquette. R. T. Taylor, Tetrahedron Lett. 1976, 2745.

"1) G. Köbrich, W. Goyert, Tetrahedron 24 (1968) 4327; D. Seyferth, R. L. Lambert jr., M. Massol, J. Organomet. Chem. 88 (1975) 255.

12) C. Herzog, R. Lang, D. Brückner, P. Kemmer, M. Christl, Chem. Ber. 119 (1986) 3027.

13) M. Christl, G. Freitag, G. Brüntrup, Chem. Ber. 111 (1978) 2307. 